\title{
SUBJECTIVE DATA REGARDING CHANGES IN GEOMETRIC FIELD OF VIEW DURING A SPEED-MATCHING TASK
}

\author{
Richard Goodenough ${ }^{1}$, Johnell Brooks ${ }^{1}$, \& Douglas Evans ${ }^{2}$ \\ ${ }^{1}$ Clemson University, Clemson, South Carolina, USA \\ ${ }^{2}$ DriveSafety, Inc., Murray, Utah, USA \\ Email: rgooden@clemson.edu
}

\begin{abstract}
Summary: One method to adjust speed perception in a driving simulator is to adjust the rendered, geometric field of view (GFOV); however, little is known regarding users' sensitivity to changing the GFOV. The current research examined 24 licensed drivers' subjective experience with changes in GFOV during a speed matching task when examining the relationship between speed estimate and GFOV in a small-footprint driving simulator. Following the completion of the speed-matching task, participants were asked three questions regarding (1) strategy used to match speed: "What strategies did you use to complete the speed matching task?”; (2) awareness of GFOV setting: "Did you notice any changes in the simulation at any time during the experiment?"; and (3) subjective accuracy: "How accurate do you think you were in performing the task on a one to ten scale, one being 'extremely inaccurate' and ten being 'extremely accurate'?” Results indicated participants were not (directly) aware of changes in the GFOV; some misattributed the change in GFOV to a change in the vehicle's acceleration rate. Furthermore, many participants' cited strategies that were later categorized as 'using optic flow' and, in general, were unsure about their accuracy in the task.
\end{abstract}

\section{INTRODUCTION}

In order for driving simulation to produce realistic experiences, users' perception of speed should correspond with real world experiences. If users are not able to perceive speed accurately, the behavior of the simulated vehicle is unlikely to match users' expectations. That is, if users are driving faster or slower than they anticipate, the simulated vehicle may react in unexpected ways. For example, a user underestimating speed may unknowingly enter a turn at an inappropriately high speed and subsequently lose control. Altering the rendered field of view, or geometric field of view (GFOV), relative to the physical, or projected field of view (PFOV), can affect users' perception of speed (e.g. Mourant, Ahmad, Jaegar, \& Lin, 2007; Diels \& Parkes, 2009). An understanding of this relationship is useful in selecting simulator display configurations to provide accurate driving experiences in a small-footprint driving simulator with a limited projected field of view $\left(65^{\circ}\right)$. The purpose of this study is to investigate the participants' subjective experience with changes in GFOV during a speed matching task used to examine the relationship between speed estimates and GFOV in a small-footprint driving simulator (See Figure 1).

Increasing the geometric field of field (GFOV) while holding the projected field of view (PFOV) constant has been shown to increase users' perceived speed (Adetiloye, Wu, \& Mourant, 2005; Mourant et al., 2007; Diels \& Parkes, 2009). In general, users underestimate their speed and 
produce higher driving speeds than intended when the veridical GFOV (GFOV/PFOV $=1.00)$ is displayed. This underestimate tends to decrease with increasing GFOV/PFOV ratios (GFOV/PFOV > 1.00), leading to smaller overproductions of speed, to a point at which the user begins to overestimate speed and produce lower driving speeds than intended.

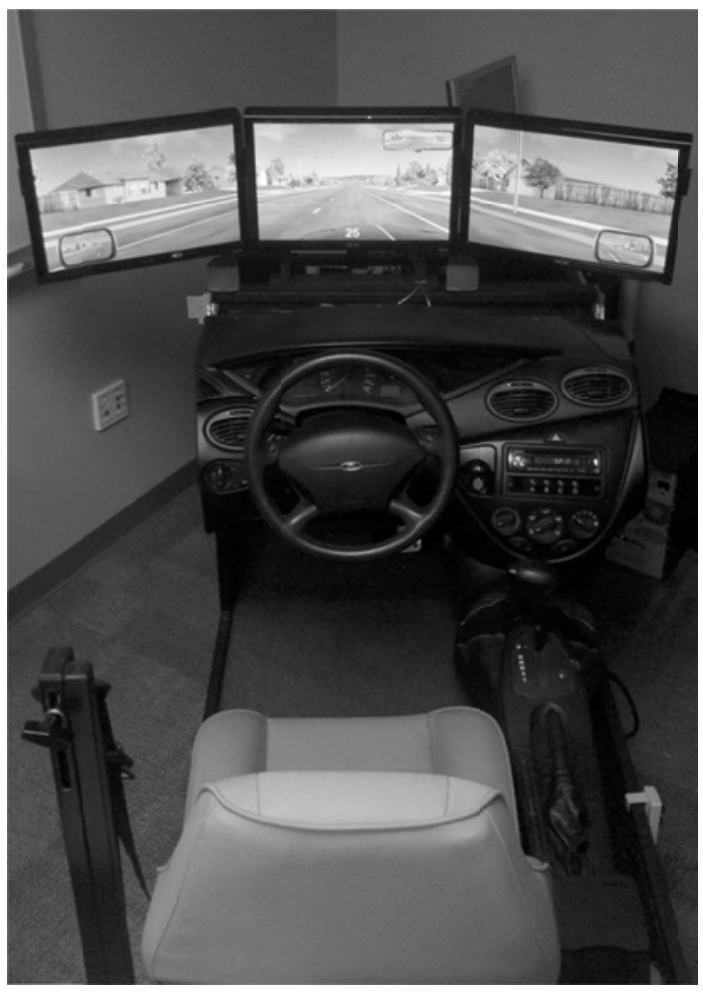

Figure 1. The DriveSafety ${ }^{\mathrm{TM}}$ CDS-250

In order to increase the GFOV/PFOV ratio beyond the veridical 1.00, a technique known as scene minification is used. Scene minification involves mathematically, in this case rectilinearly, compressing the visual scene afforded by GFOVs larger than the PFOV such that they can be presented in the same PFOV. In the case of the CDS-250, scene minification includes taking the visual scene afforded by GFOVs greater than $65^{\circ}$ and compressing it to be presented on the same display with a PFOV of $65^{\circ}$.

Increasing the GFOV/PFOV ratio has the effect of increasing the texture density in the virtual scene. This increase in texture density inherently increases the gain of optical edge rate in the virtual scene relative to longitudinal velocity. That is, increases in longitudinal velocity incur larger increases in optical edge rate. This difference between the increase in longitudinal velocity and the increase in optical edge rate becomes greater as the GFOV/PFOV ratio increases. This increase in optical edge rate should, in turn, increase the observer's perceived speed (Lee, 1979).

Diels and Parkes (2009) conducted a study investigating the effects of GFOV on speed perception. Using a large simulator with a $210^{\circ} \mathrm{PFOV}$, participants were to match a target speed (20mph, 30mph, 50mph, and 70mph) within four GFOV/PFOV ratios $(0.83,1.00,1.17$, and 1.33) without any feedback from a speedometer. Target speeds of $20 \mathrm{mph}$ and $30 \mathrm{mph}$ were presented in an urban environment, while target speeds 50mph and 70mph were presented in 
rural environment. The urban environment consisted of generic urban scenery (buildings, street lights, sidewalks, etc.), and the scenery in the rural environment consisted of fields and trees. Participants matched each target speed twice, and the mean of these two selected speeds were used in analysis. In order to compare across target speed conditions Diels and Parkes (2009) transformed the selected speeds into a ratio of selected to target speed. Similar to the results reported by previous research (e.g., Mourant et al., 2007), selected speeds decreased with increases in the GFOV/PFOV ratio. Participants tended to underestimate their speeds at lower GFOV/PFOV ratios and overestimate their speeds at higher GFOV/PFOV ratios. Five of the sixteen participants (31.25\%) said they had noticed changes in the simulation during the experiment. However, these participants all attributed what they noticed to changes in the simulated engine dynamics.

The current research included six GFOV levels including the veridical GFOV of $65^{\circ}$. Increasing by $15^{\circ}$ from the veridical, GFOVs included $65^{\circ}, 80^{\circ}, 95^{\circ}, 110^{\circ}, 125^{\circ}$, and $140^{\circ}$. These correspond to GFOV/PFOV ratios of 1.00, 1.23, 1.46, 1.69, 1.92, and 2.15, respectively. In addition, three target speeds of $25 \mathrm{mph}, 45 \mathrm{mph}$, and $65 \mathrm{mph}$ which correspond to a residential, commercial, and freeway driving environments, respectively, were examined.

\section{METHOD}

\section{Participants}

Participants included 24 licensed drivers $(11$ males, mean age $=19.4, \mathrm{SD}=1.47)$ with a minimum of 2 years of driving experience (mean years driving $=4$, SD $=1.62$ ). Each participant was screened to ensure a minimum of 20/40 high contrast visual acuity. Participants received class credit for participation.

\section{Driving Simulators}

The DriveSafety ${ }^{\mathrm{TM}}$ CDS-250 consists of a partial cab with a three-screen forward display (see Figure 1). The left and right screens are each $30^{\circ}$ off-plane from the center screen such that the center of each screen is equidistant from the driver's eyes and orthogonal to their line of sight. This produces a display measuring 49” horizontally, which yields a horizontal PFOV of $65^{\circ}$. The partial cab includes the driver's seat, dashboard, and full center console and is a reduction of the cab found in the larger DriveSafety ${ }^{\mathrm{TM}}$ DS-600. The participant controlled the vehicle using the steering wheel, accelerator, and brake. During the experimental driving sessions, the instrument cluster was covered with black felt so participants were unable to receive feedback from the speedometer.

Because practicing on the CDS-250 would require a GFOV setting to be used, there was a possibility for speed estimations to be biased toward this GFOV should it be regarded by the participant as the baseline GFOV. Therefore, participants practiced driving on a straight, twolane rural road using the DS-600 driving simulator. The DS-600 consists of a full cab surrounded by five large screens encompassing $270^{\circ}$ of view. Along with two side-view mirrors and a rearview mirror, the DS-600 provides a $360^{\circ}$ driving FOV. The DS-600 requires no visual scene compression. 


\section{Speed Matching Task}

Before each trial in the CDS-250, the participant was presented with a target speed both verbally by the experimenter and visually on the bottom-center of the center screen. The participant was told to accelerate or decelerate as much as he or she wished until satisfied the target speed had been matched. Once the participant felt the target speed had been reached, they were to press either of the two input buttons located on the steering wheel (at the '3:00' and '9:00' positions).

Target speeds included 25, 45, and 65 miles per hour. Target speed levels were chosen for their correspondence to three distinct driving environments: residential (25mph), commercial (45mph), and freeway (65mph). The target speed of 25mph was presented in a residential environment consisting of a straight, two-lane roadway lined with houses, driveways, and horticulture typically found in a residential setting. The $45 \mathrm{mph}$ target speed was presented in a commercial environment, consisting of a straight, two-lane road lined with multilevel buildings, parking lots, and their entrances. The target speed of 65mph was presented in a freeway driving environment, consisting of a 6-lane, divided road, lined intermittently with trees and shrubbery.

The GFOVs selected increased from the nominal GFOV of $65^{\circ}$ in $15^{\circ}$ increments up to $140^{\circ}$, yielding a total of six GFOV levels $\left(65^{\circ}, 80^{\circ}, 95^{\circ}, 110^{\circ}, 125^{\circ}\right.$, and $\left.140^{\circ}\right)$. These correspond to GFOV/PFOV ratios of 1.00, 1.23, 1.46, 1.69, 1.92, and 2.15, respectively. The six GFOV/PFOV ratios and three target speed levels yielded 18 experimental conditions. Each of the 18 conditions was presented to the participant three times, using a different starting point on the roadway, producing a total of 54 driving sessions per participant. The participant began each drive at one of three different start points in the environment so that a 'landmark strategy' could not be used to estimate speed between conditions. The participant was told about the different starting locations at the beginning of the experiment. The participant was allowed approximately eight minutes to complete each driving session, though each participant tended to finish a session in less than two minutes.

The order in which GFOV/PFOV ratio conditions were presented was randomized, while the target speeds were counterbalanced between participants. In attempt to reduce simulator sickness, participants were presented with all three target speeds within each GFOV/PFOV ratio condition such that the GFOV/PFOV ratio was changed only five times during an experimental session. The objective selected speed data are not discussed here.

\section{Interview Questions}

Following completion of all 54 driving sessions, the participant was asked the following three questions regarding: (1) strategy used to match speed: "What strategies did you use to complete the speed matching task?"; (2) awareness of GFOV setting: "Did you notice any changes in the simulation at any time during the experiment?"; and (3) subjective accuracy: "How accurate do you think you were in performing the task on a one to ten scale, one being 'extremely inaccurate' and ten being 'extremely accurate'?” The analysis was exploratory in nature; only descriptive statistics were calculated on these subjective data to summarize general trends. It was anticipated that the verbal descriptions of changes in the simulation as well as strategies used during the experiment would be similar enough to categorize post-hoc. 


\section{RESULTS}

\section{Strategies Used to Complete the Speed Matching Task}

Participants used one or more of three strategies: (1) 'using optic flow,' (2) 'using the simulated engine sound,' or (3) 'comparing to experience.' A participant’s strategy was categorized as 'using optic flow' if he or she mentioned using the speed of the simulated environment passing by to judge speed. For example, 'I looked at how fast stuff went by,' or 'I watched how fast the lines in the road were passing' were categorized as 'using optic flow.' Participants' strategies that included the use of any aspect of the simulated engine sound, such as 'I listened to when the gears shifted' or 'I listened to the engine RPMs,' were categorized as 'using the simulated engine sound.' Any strategy that the participant described as involving a comparison to experience, such as 'I thought about how it feels to drive at the target speed,' were categorized as 'comparing to experience.' A complete list of the statements made by the participants and how these statements were categorized into the three strategies can be found in Table 1.

Table 1. Categorization of statements made by participants regarding strategy

\begin{tabular}{|c|c|c|}
\hline $\mathrm{P}$ & Statement from participant & Categorized as \\
\hline 1 & "I looked at how fast stuff was going by; lines and signs" & Using optic flow \\
\hline 2 & "I looked at the lines in the 45 and 65 ; used the houses in the 25 " & Using optic flow \\
\hline 3 & "Watched stuff going by; listened to RPMs and shifting" & $\begin{array}{l}\text { Using optic flow / using } \\
\text { simulated engine sound }\end{array}$ \\
\hline 4 & "Watched signs going by" & Using optic flow \\
\hline 5 & "First I listened to the shifting, then started watching buildings" & $\begin{array}{l}\text { Using optic flow / using } \\
\text { simulated engine sound }\end{array}$ \\
\hline 6 & "Intuition; what I was used to; watched lines going by" & $\begin{array}{l}\text { Using optic flow / comparing } \\
\text { to experience }\end{array}$ \\
\hline 7 & "Used personal experience with how fast you should go in neighborhood, etc.” & Comparing to experience \\
\hline 8 & "How fast the lines went by" & Using optic flow \\
\hline 9 & "Watched center line, light posts, and driveways" & Using optic flow \\
\hline 10 & "Watched lines and trees" & Using optic flow \\
\hline 11 & "Tried to remember how fast lines should move at different speeds" & $\begin{array}{l}\text { Using optic flow / comparing } \\
\text { to experience }\end{array}$ \\
\hline 12 & "Used speed of objects and center line; compared to real world" & $\begin{array}{l}\text { Using optic flow / comparing } \\
\text { to experience }\end{array}$ \\
\hline 13 & "Markers on side of road; stuff going by" & Using optic flow \\
\hline 14 & "Speed of passing driveways; looked at lines on freeway" & Using optic flow \\
\hline 15 & "How fast things went by; when it shifted gears" & $\begin{array}{l}\text { Using optic flow / using } \\
\text { simulated engine sound }\end{array}$ \\
\hline 16 & “How fast peripheral stuff went by” & Using optic flow \\
\hline 17 & "Listened to engine and shifting gears" & Using simulated engine sound \\
\hline 18 & "Watched points in the distance; engine sound" & $\begin{array}{l}\text { Using optic flow / using } \\
\text { simulated engine sound }\end{array}$ \\
\hline 19 & "Watched lines on road and surroundings compared to real life" & $\begin{array}{l}\text { Using optic flow / } \\
\text { comparing to experience }\end{array}$ \\
\hline 20 & "How fast lines and surroundings were going by" & Using optic flow \\
\hline 21 & “Compared to real life; how stuff passes by” & $\begin{array}{l}\text { Using optic flow / } \\
\text { comparing to experience }\end{array}$ \\
\hline 22 & "Thought about roads I knew with those speed limits" & Comparing to experience \\
\hline 23 & "Listened to gears shifting” & Using simulated engine sound \\
\hline 24 & "Listened to gears changing; watched lines in mirrors" & $\begin{array}{l}\text { Using optic flow / using } \\
\text { simulated engine sound }\end{array}$ \\
\hline
\end{tabular}


Of the 24 participants, 10 (41.6\%) cited using strategies that were later categorized as 'using optic flow' as their only strategy, 2 (8.3\%) cited only 'using the simulated engine sound,' 2 (8.3\%) cited only 'comparing to experience,' 5 (20.8\%) cited a combination of 'using optic flow' and 'using the simulated engine sound,' and 5 (20.8\%) cited a combination of 'using optic flow' and 'comparing to experience.'

\section{Changes in Simulation Cited by Participants}

Similar to Diels and Parkes (2009), no participants conveyed in their comments they had directly noticed changes in the GFOV during the experiment. Of the 24 participants, nine (37.5\%) cited no changes in the simulation during the experiment, 14 (58.3\%) cited changes in the simulated vehicle's ability to accelerate, and one (4.2\%) cited changes in the simulated engine sound.

\section{Subjective Accuracy in Speed Matching Task}

On average, participants reported their accuracy to be 5.5 (SD $=1.38$, range: $2-8$ ) on a scale of one to ten, one being 'extremely inaccurate' and ten being 'extremely accurate'.

\section{DISCUSSION}

The purpose of the current research was to describe participants' subjective experience with changes in the GFOV in a small footprint driving simulator after completing a speed matching task. To this end, a short interview consisting of three verbal questions was conducted with each participant following the completion of all speed-matching trials.

The strategies participants cited using to complete the speed matching task point out that optic flow is often consciously used to judge speed. However, it also appears it is not the only information used. While many participants $(\mathrm{N}=10)$ cited using only the speed of the simulated environment passing by to judge their speed (later categorized as 'using optic flow'), others cited using the simulated sound of the engine $(\mathrm{N}=2)$, comparing to experience $(\mathrm{N}=2)$, or a combination of using optic flow and either the simulated engine sound $(\mathrm{N}=5)$ or comparing to experience $(\mathrm{N}=5)$. The fact that participants cited using the simulated engine sound as part of their strategy suggests that muting the sound would have also limited extraneous speed information.

The subjective data regarding whether or not participants noticed the changes in the simulation during the experimental trials indicates that no participant directly identified changes in the GFOV. This is likely due to the fact participants never consecutively drove through the same simulated environment using different GFOV/PFOV ratios, limiting their ability to easily make direct comparisons between GFOV/PFOV ratio conditions. Participants also began each drive at different points along the roadway. Because participants were made aware of these different starting points before beginning the experimental sessions, they may have attributed the changes they saw in the display to changes in their starting location. 
Moreover, participants who reported they did notice a change credited it to differences in the simulated vehicle's ability to accelerate. This is what one would expect to find with changes in the gain of optic flow relative to simulated vehicle velocity. That is, the increase in the velocity of optic flow becomes larger compared to the increase in the simulated vehicle velocity as the GFOV/PFOV ratio increases. This gain in optic flow, therefore, provides illusory visual information that the simulated vehicle is accelerating at a faster rate at higher GFOV/PFOV ratios compared to lower ratios. This also suggests that participants were attending to the simulated vehicle's acceleration rates during the experiment. Future research may consider altering the simulated vehicle's dynamics such that acceleration rates were constant regardless of velocity. This may have helped to limit the speed information participants were able to receive through the simulated vehicle's dynamics rather than through only the optic flow.

The participants' estimates regarding how accurate they were in the speed matching task emphasize some important characteristics of the task itself. The mean rating of 5.5 on a one to ten scale, one being 'extremely inaccurate' and ten 'extremely accurate,' suggests that participants were, in general, unsure how well they performed. This could be because participants received no feedback regarding the accuracy of their speed estimates. These subjective accuracy ratings also highlight the general difficulty of estimating one's speed in a driving simulator without feedback from the speedometer (Hurwitz, Knodler, \& Dulaski, 2005; Kemeny \& Panerai, 2003), further emphasizing the importance of ensuring the selected GFOV supports accurate speed perception.

\section{REFERENCES}

Adetiloye, C., Wu, Q., \& Mourant, R. R. (2005). Perception of optical flow and geometric field of view. Proc. 32nd International Conference on Computer Graphics and Interactive Techniques (SIGGRAPH 2005).

Diels, C., \& Parkes, A. M. (2009). Geometric field of view manipulations affect perceived speed in driving simulators. Proc. International Conference of Road Safety and Simulation (RSS 2009). Paris, France.

Hurwitz, D. S., Knodler, M. A., Dulaski, \& D. M. (2005). Speed perception fidelity in a driving simulator environment. DSC 2005 North America. Orlando, FL.

Kemeny, A., \& Panerai, F. (2003). Evaluating perception in driving simulation experiments. Trends in Cognitive Sciences, 7(1), 31-37.

Lee, D. N. (1979). Visual information during locomotion. In R. MacLeod, \& H. Pick (Eds.), Perception: Essays in Honor of James J. Gibson (pp. 250-267). Ithaca, NY: Cornell University Press.

Mourant, R. R., Ahmad, N., Jaeger, B. K., \& Lin, Y. (2007). Optic flow and geometric field of view in a driving simulator display. Displays, 145-149. 